\title{
Influencia de la vigilancia en una zona rocosa dentro de un área marina protegida, Pacífico Central de Costa Rica
}

\author{
Juan Carlos Azofeifa-Solano ${ }^{1,2^{*}}$, Carolina Salas-Moya ${ }^{2,3}$, Yamileth Cubero-Campos ${ }^{4}$ \\ \& Jeffrey A. Sibaja-Cordero ${ }^{2,3}$ \\ 1. Programa de Maestría en Biología, Sistema de Estudios de Posgrado, Universidad de Costa Rica, San Pedro, 11501- \\ 2060 San José, Costa Rica; juan.azofeifa@ucr.ac.cr \\ 2. Centro de Investigación en Ciencias del Mar y Limnología (CIMAR), Universidad de Costa Rica, San Pedro, 11501- \\ 2060 San José, Costa Rica; carosalasm@gmail.com, jeffro.alejandro@gmail.com \\ 3. Escuela de Biología, Universidad de Costa Rica, San Pedro, 11501-2060 San José, Costa Rica. \\ 4. Área de Conservación Pacífico Central, Sistema Nacional de Áreas de Conservación, Costa Rica; \\ yamileth.cubero@sinac.go.cr
}

Recibido 21-II-2018. C Corregido 30-IV-2018. Aceptado 07-VI-2018.

\begin{abstract}
Surveillance influence in a rocky shore within a marine protected area, Central Pacific of Costa Rica. Rocky intertidal are important sites for fish, birds, invertebrates and algae, among others, which are used for the extraction of food resources for coastal communities. We present the results of conservation assessment of a rocky shore in the Central Pacific of Costa Rica (Playa Hermosa-Punta Mala National Wildlife Refuge) using the biological indicators proposed by the National Environmental Ministry. This study aims to analyze the influence of surveillance, between two sites, over the rocky shore habitat. The sampling was carried out during both the dry and rainy seasons in 2015. Our data shows that Nerita scabricosta -harvested in small-scale fisheries- presents a high number of colonies formed by few individuals. Moreover, the abundance varied between seasons, probably due to a storm surge. Macroalgae differed slightly among sites, where turf had higher percentage coverage in low surveillance areas, while brown algae had higher coverage in high surveillance areas. Mechanical damage (abrasion due to rocks overturning) associated with tourism and harvesting could be influencing the low surveillance area, explaining these differences. Macroalgae presented a seasonal change, probably related to the storm surge. The density of the Tetraclita stalactifera did not exhibit variations. The surveillance level and enforcement influenced some bioindicators of the rocky shore, and therefore could be used to assess the extractive pressure. Rev. Biol. Trop. 66(3): 984-995. Epub 2018 September 01.
\end{abstract}

Key words: bioindicator; Costa Rican coastal zone; intertidal macroalgae; marine protected área; Nerita scabricosta; Punta Mala; Tetraclita stalactifera.

Las costas rocosas están presentes en todas las líneas costeras de los océanos, desde los polos hasta los trópicos. Estos hábitats son sitios de reclutamiento para peces e invertebrados que viven como fauna críptica, epifauna móvil y sésil (Marliave, 1986; Menge, 1991) y presentan gran diversidad de micro y macroalgas (Crowe, Thompson, Bray, \& Hawkins, 2000; Little \& Kitching, 1996). Los intermareales rocosos son sitios de alimentación para aves en marea baja, y para peces y plancton durante la marea alta (Barrantes \& Pereira, 1992; Mazé, Fuertes, \& Pena, 2006; Ribeiro, Iribarne, Navarro, \& Jaureguy, 2004; Withers \& Chapman, 1993). Además, son económicamente importantes para las comunidades costeras ya que se utilizan para la extracción de recursos alimenticios (Riera et al., 2016; Rojas, Villalobos, Chartier, \& Villalobos, 1988). A pesar de su importancia, muchos litorales rocosos se han visto afectados por actividades humanas, tales como la presión pesquera, modificación de la 
costa, sedimentación y contaminación (Crowe et al., 2000; Davenport \& Davenport, 2006).

El establecimiento de Áreas Marinas Protegidas (AMP) puede minimizar los impactos humanos sobre los organismos marinos, e incluso ayudar a restablecer las poblaciones y los paisajes naturales (Casu, Ceccherelli, Curini-Galletti, \& Castelli, 2006; Lester et al., 2009). Sin embargo, el efecto de las AMP no es siempre el esperado, ya que pueden ocurrir cambios positivos o negativos en las poblaciones o comunidades marinas, o bien el efecto de recuperación puede ocurrir lentamente o rápidamente, dependiendo del impacto que se da alrededor o previo al establecimiento del AMP (Lester et al., 2009). Por otro lado, algunas AMP requieren una vigilancia eficiente para lograr implementar las medidas de conservación (Alvarado, Cortés, Esquivel, \& Salas, 2012). Por lo tanto, el monitoreo de las AMP es indispensable para verificar su efectividad y facilitar la toma de decisiones en los esfuerzos de conservación y manejo (Banks \& Skilleter, 2002; Murray, Ambrose, \& Dethier, 2006).

Actualmente están ocurriendo cambios ambientales de manera acelerada debido a la influencia humana, por lo que es necesario contar con indicadores clave que brinden una idea del estado de los ecosistemas en poco tiempo, para poder tomar decisiones de manejo de forma eficiente (Defeo et al., 2009; SibajaCordero, Camacho-García, \& Vargas-Castillo, 2014). Las costas rocosas presentan especies que pueden ser usadas como indicadores de la presión humana, ya sea por su presencia, talla o porque son utilizadas como alimento (Murray et al., 2006). El objetivo de este trabajo es analizar la influencia de la vigilancia (patrullaje de los guarda parques) sobre el hábitat intermareal rocoso dentro de un AMP, utilizando los bioindicadores del protocolo de costas rocosas del Sistema Nacional de Áreas de Conservación de Costa Rica (Sibaja-Cordero \& MartínezFernández, 2016). Los resultados del presente estudio se pueden utilizar como línea base para dirigir la gestión del AMP, de modo que se puedan detectar las posibles causas que provocan el deterioro de estas comunidades biológicas.

\section{MATERIALES Y MÉTODOS}

Área de estudio: El Refugio Nacional de Vida Silvestre Playa Hermosa-Punta Mala (RNVS-PHPM) fue creado mediante el Decreto Ejecutivo N-27 210, del 16 de abril de 1998, para la conservación de la tortuga lora, Lepidochelys olivacea Eschscholtz, 1829, y se amplió para la protección de otras especies marinas y ecosistemas, mediante el Decreto N-33 044, del 03 de mayo 2002. El RNVS-PHPM se ubica en el Pacífico Central de Costa Rica ( $9^{\circ} 31^{\prime}$ N \& $84^{\circ} 32^{\prime} \mathrm{W}$ ) y tiene una extensión de 2742 hectáreas (ha), donde $82 \%$ (2 247 ha) son marinas y $18 \%$ (495 ha) son playas, humedales y una pequeña extensión de bosque detrás de la playa. En esta área la época seca se extiende de diciembre a abril, mientras que la época lluviosa abarca de mayo a noviembre (Sutherland \& Ortega, 1986). En la parte marina, son frecuentes los vientos del suroeste con velocidad promedio de $11.5 \mathrm{~km} / \mathrm{h}$, y ráfagas de hasta 45.25 $\mathrm{km} / \mathrm{h}$, la olas del suroeste son frecuentes, con altura promedio de $1.33 \mathrm{~m}$ y máximas de 2.81 $\mathrm{m}$, la temperatura promedio del agua es 28.45 ${ }^{\circ} \mathrm{C}$ con una salinidad de $31.86 \mathrm{psu}$, la marea es del tipo semidiurna (ciclos $\sim 12$ horas), con una marea promedio de $1.40 \mathrm{~m}$ y máxima de $3.30 \mathrm{~m}$ (Lizano, 2006; MIO-CIMAR, 2017).

Punta Mala, también conocida como Punta Judas (9॰30'51.81” N \& 84³2'5.38” W), es un sistema rocoso dentro del RNVS-PHPM (Fig. 1). El promontorio rocoso se prolonga horizontalmente hacia el sur, con un buzamiento que varía entre los $5^{\circ}$ y $30^{\circ}$ (Aguilar, 1998; Sutherland \& Ortega, 1986), que se extiende hacia el mar y el pueblo de Esterillos Oeste, sobrepasa los límites actuales del RNVS-PHPM y forma una plataforma de $4 \mathrm{~km}$ de largo y hasta $1 \mathrm{~km}$ de ancho. Además, está conformado por areniscas líticas y otras rocas clásticas de origen somero (Aguilar, 1998; Seyfried, Sprechmann, \& Aguilar, 1985; Ugalde, 2012). En dicha zona se pueden encontrar varios moluscos que son extraídos para consumo humano (Ortega, 1987).

Diseño de muestreo: El estudio se realizó en la plataforma intermareal rocosa de Punta 


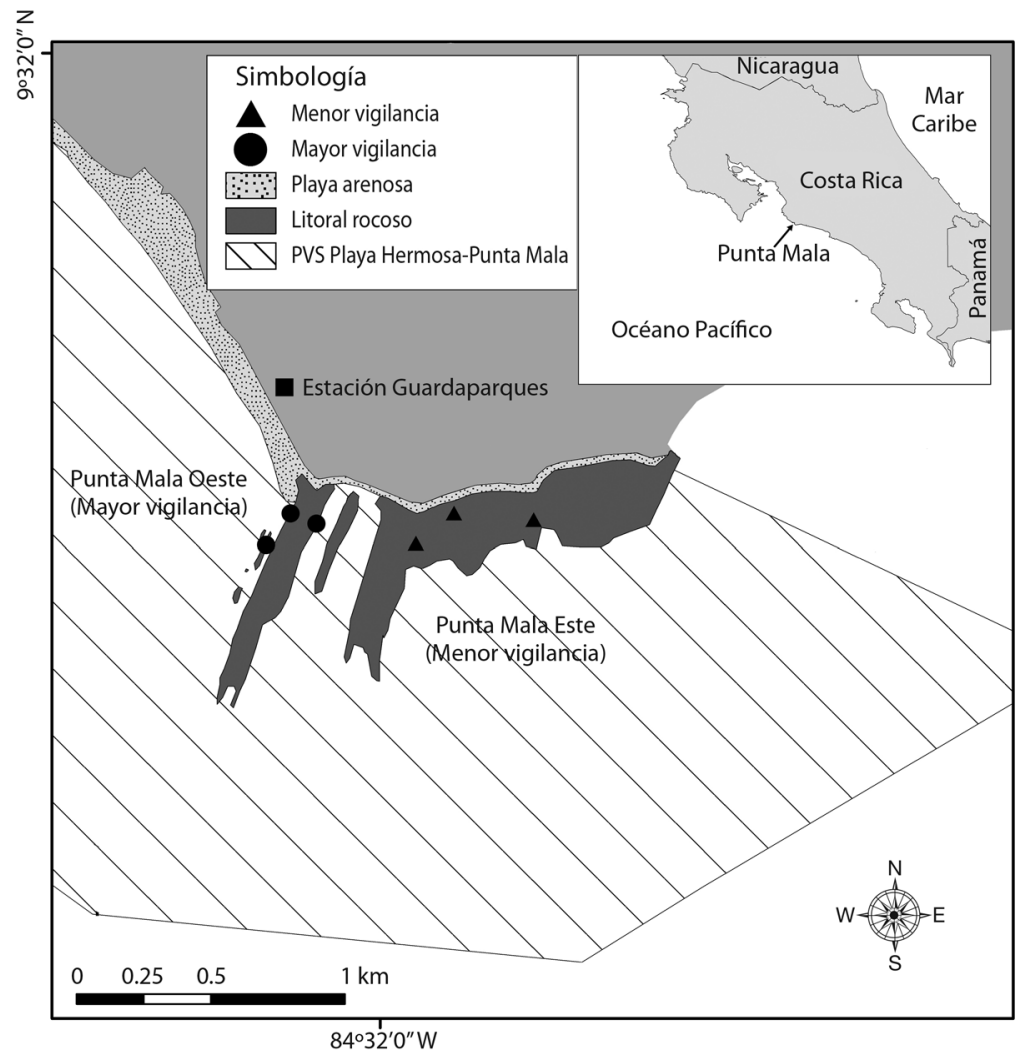

Fig. 1. Zonas de monitoreo en la costa rocosa según nivel de protección del Refugio Nacional de Vida Silvestre Playa Hermosa-Punta Mala, Pacífico de Costa Rica (2015).

Fig. 1. Sampling zones in the rocky shore by level of protection in the National Wildlife Refuge Playa Hermosa-Punta Mala, Costa Rican Pacific (2015).

Mala, donde se establecieron dos zonas de recolecta según el nivel de vigilancia por parte de los guarda parques: 1) Mayor vigilancia, hacia el oeste de Punta Mala y frente al puesto de guardaparques del Refugio; 2) Menor vigilancia, hacia el este de Punta Mala, fuera del rango de visión del puesto de guarda parques del Refugio y cerca del poblado Esterillos (Fig. 1, área con mayor vigilancia: 9³1'04.36" $\mathrm{N}$ \& 84 32'10.8" $\mathrm{W}$, área con menor vigilancia: 9॰31'04.4" N \& 84³1'51.1” W). La distinción se basó en la información suministrada por los mismos guardaparques del Refugio. Las dos zonas se visitaron caminando en horas de la mañana y durante la bajamar en tiempo de mareas vivas, el 26-27 de febrero (época seca) y el 21-22 de mayo 2015 (inicio de la época lluviosa). Con el fin de analizar la influencia de la vigilancia sobre el intermareal rocoso, se seleccionaron como indicadores la cantidad de agregaciones de Nerita scabricosta Lamarck, 1822, la densidad de Tetraclita stalactifera (Lamarck, 1818), la composición de grupos funcionales de macroalgas y grupos taxonómicos superiores de invertebrados bajo las rocas. La metodología para cada uno de estos indicadores se indica en el cuadro 1. En cada sitio (con mayor vigilancia y con menor vigilancia) y durante cada visita (época seca y lluviosa) se establecieron tres estaciones (réplicas) en las que se ejecutó el protocolo de monitoreo, detallado en Sibaja-Cordero y Martínez-Fernández (2016). 
CUADRO 1

Indicadores biológicos utilizados en el monitoreo de una costa rocosa, Punta Mala, Pacífico de Costa Rica (2015), basado en Sibaja-Cordero \& Martínez-Fernández (2016)

TABLE 1

Biological indicators used in the survey of a rocky shore, Punta Mala, Costa Rican Pacific (2015), from Sibaja-Cordero \& Martínez-Fernández (2016)

\begin{tabular}{|c|c|c|}
\hline Especie o grupo & Indicador & Metodología \\
\hline Nerita scabricosta & $\begin{array}{l}\text { Cantidad de } \\
\text { agrupaciones }\end{array}$ & $\begin{array}{l}\text { Se observaron las agrupaciones de } N \text {. scabricosta a lo largo del } \\
\text { supralitoral, en tres transectos de } 20 \mathrm{~m} \text { largo } \times 2 \mathrm{~m} \text { ancho. Las } \\
\text { agregaciones se clasificaron y contabilizaron según la abundancia de } \\
\text { individuos: alta }(>20) \text {, media }(10-20) \text { y baja }(<10) \text {. }\end{array}$ \\
\hline Tetraclita stalactifera & $\begin{array}{l}\text { Densidad de } \\
\text { individuos }\end{array}$ & $\begin{array}{l}\text { Se realizaron tres transectos de } 20 \mathrm{~m} \text { en el litoral bajo, en cada uno se } \\
\text { colocaron } 10 \text { cuadrículas }(25 \times 25 \mathrm{~cm}) \text { cada } 2 \mathrm{~m} \text {. En cada cuadrícula } \\
\text { se cuantificaron los individuos vivos de } T \text {. stalactifera. }\end{array}$ \\
\hline Macroalgas intermareales & $\begin{array}{l}\text { Composición de } \\
\text { grupos funcionales }\end{array}$ & $\begin{array}{l}\text { Se realizaron tres transectos de } 20 \mathrm{~m} \text { en el infralitoral, en cada uno } \\
\text { se colocaron } 10 \text { cuadrículas }(50 \times 50 \mathrm{~cm} \text { ), subdivididas cada } 10 \\
\mathrm{cm} \text {, y espaciadas ente sí cada } 2 \mathrm{~m} \text {. En la cuadrícula se cuantificó } \\
\text { la cobertura de algas sobre el sustrato utilizando las siguientes } \\
\text { categorías: foliosa verde (e.g. Halimeda sp., Ulva sp.), foliosa roja } \\
\text { (e.g. Laurencia } \text { sp.), foliosa parda (e.g. Padina sp.), incrustante } \\
\text { calcárea, y tapete de algas (conocido como “turf"). }\end{array}$ \\
\hline Invertebrados bajo las rocas & $\begin{array}{l}\text { Presencia de grupos } \\
\text { taxonómicos } \\
\text { superiores }\end{array}$ & $\begin{array}{l}\text { En cada sitio se voltearon azarosamente } 45 \text { cantos rodados en el } \\
\text { infralitoral. Para cada roca se anotó la presencia de la fauna móvil } \\
\text { y sésil habitando debajo. Después de tomar los datos, cada roca fue } \\
\text { volteada nuevamente y colocada en el sitio donde se encontró. }\end{array}$ \\
\hline
\end{tabular}

Agrupaciones de Nerita scabricosta: para determinar cuáles factores influyeron sobre la cantidad de agrupaciones de N. scabricosta, se realizó un modelo simplificado tipo "loglinear" con distribución Poisson utilizando la librería Mass (Venables \& Ripley, 2013) en el paquete estadístico RStudio v 0.99.484 (RStudio Team, 2015), siguiendo los pasos descritos en Crawley (2007). Los factores del modelo saturado fueron el "nivel de vigilancia" (Mayor vigilancia, Menor vigilancia), la época (seca, lluviosa), la abundancia de individuos en las agrupaciones (baja, media, alta) y todas las posibles interacciones de estos.

Densidad de Tetraclita stalactifera: se realizó un Análisis de Varianza (ANDEVA) de dos vías, para determinar si la densidad (individuos $/ \mathrm{m}^{2}$ ) de T. stalactifera varió, considerando como factores el nivel de vigilancia y la época, utilizando PAST v 3.08 (Hammer, Harper, \& Ryan, 2001). Los datos de densidad se transformaron utilizando raíz cuadrada para cumplir los requisitos de homogeneidad de varianza y normalidad de residuales del ANDEVA (Krebs, 1999).

Macroalgas intermareales: el porcentaje de cobertura de cada grupo de macroalgas se trasformó utilizando la función arcoseno (Krebs, 1999). Para determinar el patrón de la composición de macroalgas según el nivel de vigilancia y la época, se realizaron un análisis de escalamiento multidimensional (MDS, por sus siglas en inglés) utilizando distancia euclidiana, y un análisis de similitudes (ANOSIM) con 9999 permutaciones, utilizando PAST (Hammer et al., 2001). Se aplicó un análisis de porcentaje de similitudes (SIMPER) para determinar cómo los grupos de macroalgas contribuyeron a las diferencias entre los sitios y épocas, utilizando PRIMER 6.0 (Clarke \& Gorley, 2006).

Invertebrados bajo las rocas: para determinar el patrón de presencia de invertebrados 
según el nivel de vigilancia y la época, se realizaron un MDS utilizando el índice de Dice (Sorensen), y un ANOSIM utilizando PAST (Hammer et al., 2001). Se realizó un SIMPER para determinar cómo los grupos de invertebrados aportan en la disimilitud según los sitios y épocas, utilizando PRIMER 6.0 (Clarke \& Gorley, 2006).

\section{RESULTADOS}

Agrupaciones de Nerita scabricosta: Los resultados del modelo "log-linear" mostraron que la "época" (seca, lluviosa) y el "nivel de vigilancia" (menor vigilancia, mayor vigilancia) influenciaron la cantidad de agregaciones de N. scabricosta (Cuadro 2). En general hubo más agrupaciones en el sitio con menor vigilancia que en el sitio con mayor vigilancia (Fig. 2; Cuadro 2; $\mathrm{P}<0.001$ ). El número de agrupaciones en el sitio con menor vigilancia fue muy alto durante la época seca, pero disminuyó durante la época lluviosa (Fig. 2; Cuadro 2; $\mathrm{P}<0.001)$.

En general las agrupaciones de abundancia baja fueron más comunes que las agrupaciones de abundancia media y alta juntas (Fig. 2). La cantidad de agrupaciones según abundancia varió entre los niveles de vigilancia (Fig. 2; Cuadro 2; $\mathrm{P}<0.001)$. Durante la época lluviosa en el sitio con mayor vigilancia se encontraron más agrupaciones de abundancia alta que durante la época seca, mientras que las agrupaciones de baja abundancia fueron más comunes en el sitio de menor vigilancia (Fig. 2; Cuadro 2; $\mathrm{P}<0.001$ ).

Densidad de Tetraclita stalactifera: La densidad de T. stalactifera varió entre 16 y $1216 \mathrm{ind} / \mathrm{m}^{2}$ con un promedio $( \pm \mathrm{DE}) \mathrm{de}$ $320 \pm 38 \mathrm{ind} / \mathrm{m}^{2}$. El promedio de densidad de ind $/ \mathrm{m}^{2}$ resultó similar entre los niveles de

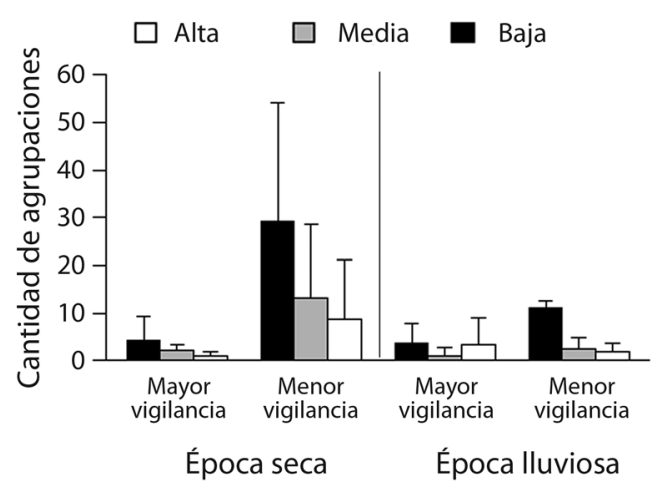

Fig. 2. Promedio del número agrupaciones ( $\pm \mathrm{DE}$ ) de Nerita scabricosta en una costa rocosa tropical según el nivel de vigilancia y la época, Pacífico de Costa Rica (2015). Negro $=$ baja abundancia, Gris = media abundancia, Blanca $=$ alta abundancia.

Fig. 2. Average of numbers of groups $( \pm \mathrm{SD})$ of Nerita scabricosta in a tropical rocky shore by surveillance level and season, Costa Rican Pacific (2015). Black = low abundance, Gray = medium abundance, White = high abundance.

\section{CUADRO 2}

Modelo linear-log con distribución Poisson usado para examinar el efecto del nivel de vigilancia sobre la presencia de agrupaciones de Nerita sacbricosta en una costa rocosa tropical, Pacífico de Costa Rica (2015)

TABLE 2

Log-linear model with Poisson distribution used to test the effect of surveillance level on the groups of Nerita scabricosta in a rocky shore of Costa Rican Pacific (2015)

\begin{tabular}{lccccc} 
& $\begin{array}{c}\text { Grados de } \\
\text { libertad }\end{array}$ & Desviación & $\begin{array}{c}\text { Grados de libertad de } \\
\text { los residuales }\end{array}$ & $\begin{array}{c}\text { Desviación de } \\
\text { los residuales }\end{array}$ & $\begin{array}{c}\text { Probabilidad } \\
\text { Modelo Saturado }\end{array}$ \\
Vigilancia & - & - & 35 & 412.02 & - \\
Época & 1 & 102.98 & 34 & 309.04 & $<0.001$ \\
Abundancia & 1 & 46.79 & 33 & 262.24 & $<0.001$ \\
Vigilancia: Abundancia & 2 & 67.12 & 31 & 195.12 & $<0.001$ \\
\hline
\end{tabular}

Modelo saturado: Frecuencia de agregaciones $\sim$ Nivel de vigilancia + Época + Abundancia de las agregaciones + Nivel de vigilancia $\times$ Época $\times$ Abundancia de las agregaciones. 


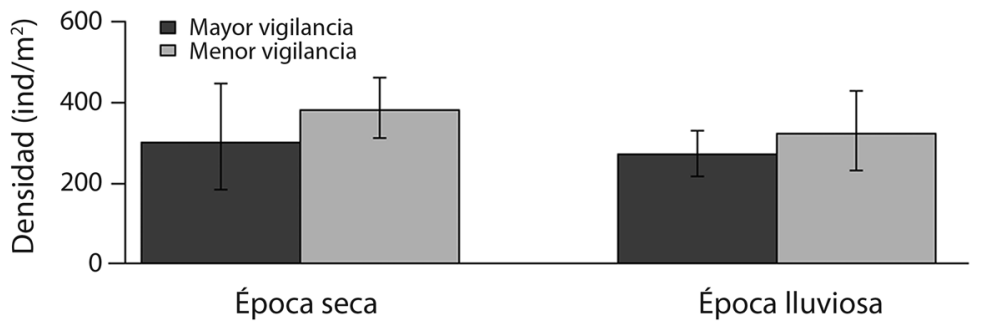

Fig. 3. Densidad promedio ( $\pm \mathrm{DE})$ de Tetraclita stalactifera en una costa rocosa tropical según el nivel de vigilancia y la época, Pacífico de Costa Rica (2015).

Fig. 3. Mean density $( \pm \mathrm{SD})$ of Tetraclita stalactifera in a tropical rocky littoral by surveillance level and season, Costa Rican Pacific (2015).

vigilancia $(\mathrm{F}=3.01 ; \mathrm{gl}=1 / 102 ; \mathrm{P}=0.086)$ $\mathrm{y}$ entre las épocas $(\mathrm{F}=1.10 ; \mathrm{gl}=1 / 102 ; \mathrm{P}=$ 0.130) (Fig. 3).

Macroalgas intermareales: Durante la recolecta de datos, se observaron parches de tapete de algas y algas incrustantes calcáreas, junto con algunos pocos talos de Dyctiota sp. (foliosa parda) y Halimeda sp. (foliosa verde). El tapete de algas fue el grupo de macroalgas más común, presente en $74 \%$ de las cuadrículas, seguido de las foliosas pardas (61\%) y las algas incrustantes calcáreas (39\%). El tapete de algas cubrió en promedio $52 \%$ del área, seguido de las foliosas pardas (16\%). Las foliosas verdes aparecieron solamente en $6 \%$ de las cuadrículas y cubrieron en promedio 0.2 $\%$. Se observó una tendencia a la separación entre los niveles de vigilancia según la composición de macroalgas intermareales (ANOSIM, $\mathrm{R}=0.24 ; \mathrm{P}<0.001)$. En el MDS los cuadrantes del sitio con menor vigilancia se ubican hacia valores positivos de la Dimensión 2 y los de mayor vigilancia se ubican hacia valores negativos de la Dimensión 2 (Fig. 4A). En el mismo sentido, se encontró que para ambos niveles de vigilancia, las estaciones de la época seca (izquierda de MDS en Fig. 4B y Fig. 4C) y

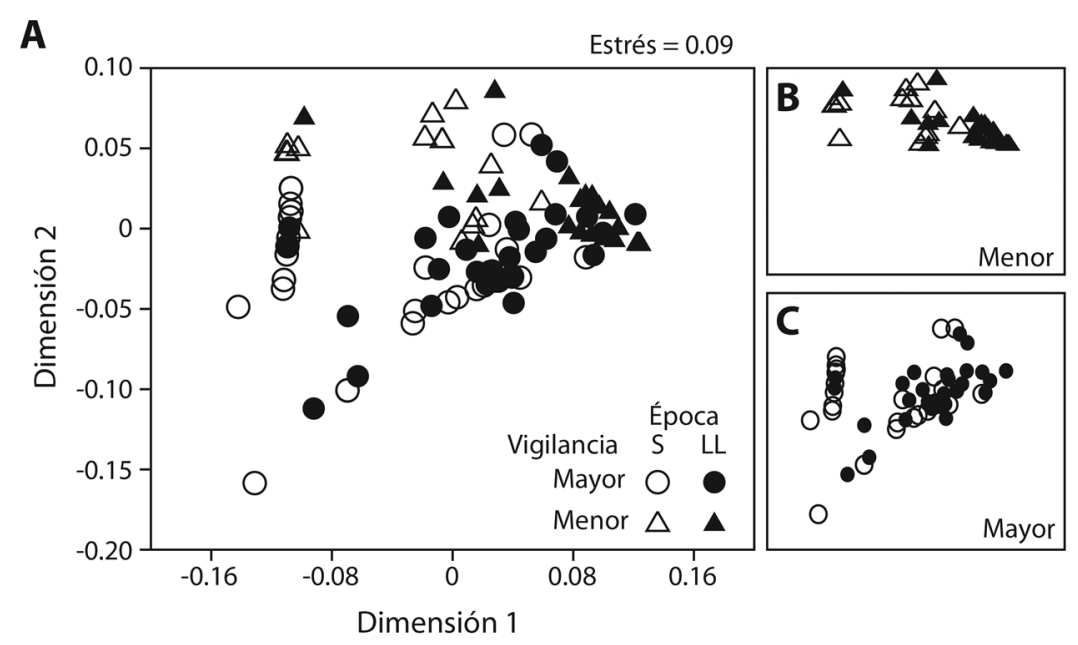

Fig. 4. A. Análisis escalado multidimensional (MDS) para mostrar la similitud de los sitios y las épocas según la composición de las algas intermareales y subset para las cuadriculas de B. menor vigilancia y $\mathbf{C}$. mayor vigilancia, en una costa rocosa tropical, Pacífico de Costa Rica (2015). S= época seca, LL=época lluviosa.

Fig. 4. A. Multidimensional Scaling Analysis (MDS) to show the similarity of the sites by seasons according to the composition of the intertidal algae and subset for the B. lower surveillance and $\mathbf{C}$. higher surveillance, on a tropical rocky coast, Costa Rica (2015). S = dry season, LL = rainy season. 
época lluviosa (derecha del MDS en Fig. 4B y Fig. 4C) se separaron por la composición de los grupos funcionales de macroalgas (ANOSIM, $\mathrm{R}=0.42 ; \mathrm{P}<0.001)$. A partir del SIMPER se mostró que el tapete de algas, las foliosas pardas y las incrustantes calcáreas, contribuyeron en ese orden a la variación de la composición, tanto para el factor "época" como el "nivel de vigilancia” (Cuadro 3).

Invertebrados bajo las rocas: El grupo de fauna más frecuente bajo las rocas fue Ophiuroidea, seguido por Gastropoda, Porifera y Crustacea (presentes en más del $40 \%$ de los cantos). Otros grupos comunes fueron: Polychaeta, Bryozoa y Holothuroidea (presentes entre $40 \%$ y $20 \%$ de los cantos). Los grupos menos frecuentes $(<20 \%)$ en los cantos fueron: Polyplacophora, Anthozoa y Echinoidea. El MDS no mostró una separación entre las muestras (Fig. 5), pero el ANOSIM demostró que la composición de la fauna varió levemente según el nivel de vigilancia $(\mathrm{R}=$ 0.10; $\mathrm{P}<0.001$ ), donde Ophiuroidea, Bryozoa y Holothuroidea ocurrieron con mayor frecuencia en el sitio de mayor vigilancia, mientras que Crustacea, Porifera, Gastropoda y Polychaeta se presentaron con mayor frecuencia en la zona de menor vigilancia. Por otro lado la composición de la fauna no varió según la época $(\mathrm{R}=$
0.04; $\mathrm{P}=0.05)$. El cuadro 4 muestra los grupos de invertebrados que más aportaron en la disimilitud según el nivel de vigilancia.

\section{DISCUSIÓN}

Agrupaciones de Nerita scabricosta: Durante 1984 no se reportó $N$. scabricosta en Punta Mala (previo al establecimiento del

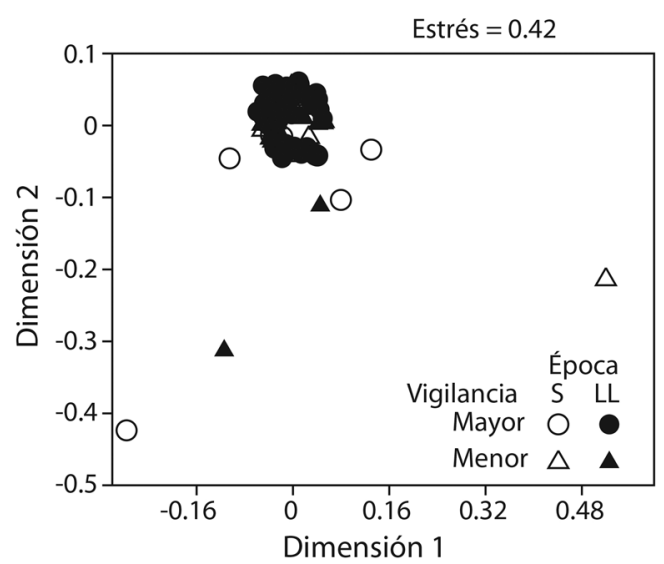

Fig. 5. Análisis escalado multidimensional (MDS) para mostrar la similitud de las estaciones según la fauna que habita bajo los cantos rodados, en una costa rocosa tropical, Pacífico de Costa Rica (2015). Fig. 5. Multidimensional scaling analysis (MDS) to show the similarity of the stations according to the fauna that lives under the pebbles, in a tropical rocky coast, Pacific of Costa Rica (2015).

\section{CUADRO 3}

Porcentajes de contribución a la disimilitud (SIMPER) de la cobertura de algas según el nivel de vigilancia y la época en una costa rocosa tropical, Pacífico de Costa Rica (2015)

TABLE 3

Percentages of contribution to the disimilarity (SIMPER) of the algal cover by surveillance level and season in a tropical rocky shore, Costa Rican Pacific (2015)

\begin{tabular}{lcccc}
\multicolumn{1}{c}{$\begin{array}{c}\text { Alga: } \\
\text { Factor vigilancia }\end{array}$} & $\begin{array}{c}\text { Porcentaje de } \\
\text { contribución }\end{array}$ & $\begin{array}{c}\text { Porcentaje } \\
\text { acumulado }\end{array}$ & $\begin{array}{c}\text { Promedio de cobertura } \\
\text { en mayor vigilancia }\end{array}$ & $\begin{array}{c}\text { Promedio de cobertura } \\
\text { en menor vigilancia }\end{array}$ \\
Tapete de algas & 60.94 & 60.94 & 0.69 & $\mathbf{0 . 8 5}$ \\
Foliosas pardas & 28.81 & 89.75 & $\mathbf{0 . 5 0}$ & 0.10 \\
Incrustantes costrosas & 8.85 & 98.60 & 0.09 & $\mathbf{0 . 1 7}$ \\
\multicolumn{1}{c}{ Factor época } & Porcentaje de & Porcentaje & Promedio de cobertura & Promedio de cobertura \\
contribución & acumulado & en época seca & 0.42 & $\mathbf{1 . 0 9}$ \\
Tapete de algas & 71.36 & 71.36 & $\mathbf{0 . 1 4}$ & 0.12 \\
Foliosas pardas & 20.11 & 91.47 & $\mathbf{0 . 4 1}$ & 0.23 \\
Incrustantes costrosas & 7.30 & 98.76 & & \\
\hline
\end{tabular}


CUADRO 4

Porcentajes de contribución a la disimilitud (SIMPER) de la fauna que habita bajo los cantos según el nivel de vigilancia y la época en una costa rocosa tropical, Pacífico de Costa Rica (2015)

TABLE 4

Percentage of contribution to the disimilarity (SIMPER) of the fauna that life under the pebbles by surveillance level and season in a tropical rocky shore, Costa Rican Pacific (2015)

\begin{tabular}{lcccc}
\multicolumn{1}{c}{$\begin{array}{c}\text { Fauna: } \\
\text { Factor vigillancia }\end{array}$} & $\begin{array}{c}\text { Porcentaje de } \\
\text { contribución }\end{array}$ & $\begin{array}{c}\text { Porcentaje } \\
\text { acumulado }\end{array}$ & $\begin{array}{c}\text { Promedio de ocurrencia en } \\
\text { mayor vigilancia }\end{array}$ & $\begin{array}{c}\text { Promedio de ocurrencia } \\
\text { en menor vigilancia }\end{array}$ \\
Crustacea & 13.68 & 13.68 & 0.26 & $\mathbf{0 . 5 4}$ \\
Ophiuroidea & 13.40 & 27.08 & $\mathbf{0 . 6 7}$ & 0.47 \\
Porifera & 13.27 & 40.35 & 0.35 & $\mathbf{0 . 5 1}$ \\
Gastropoda & 13.15 & 53.50 & 0.26 & $\mathbf{0 . 5 0}$ \\
Polychaeta & 12.40 & 65.90 & 0.15 & $\mathbf{0 . 3 7}$ \\
Briozoa & 10.85 & 76.76 & $\mathbf{0 . 3 5}$ & 0.21 \\
Holothuroidea & 10.03 & 86.79 & $\mathbf{0 . 3 2}$ & 0.17 \\
Opistobranchia & 5.34 & 92.13 & 0.03 & $\mathbf{0 . 1 9}$ \\
Polyplacophora & 4.28 & 96.41 & $\mathbf{0 . 1 5}$ & 0.01 \\
\hline
\end{tabular}

RNVS-PHPM), y su ausencia se relacionó con el exceso de extracción en años previos (Ortega, 1987). Sin embargo, durante 2015 fue común observar $N$. scabricosta en el supralitoral rocoso (248 agrupaciones, Fig. 2). Es posible que la vigilancia y protección de la zona costera permitiera el re-establecimiento de poblaciones de $N$. scabricosta. Un proceso similar se observó en el Parque Nacional Manuel Antonio (Pacífico Central de Costa Rica), donde no se reportó Siphonaria gigas (utilizada para consumo humano en la zona) en 1962, debido posiblemente a la extracción pesquera, y luego fue observada en 1995 después del establecimiento del Parque Nacional en 1972 (Bakus, 1968; Ortega, 1987; Willis \& Cortés, 2001). La reaparición de S. gigas en el Parque Nacional Manuel Antonio y de $N$. scabricosta en el RNVS-PHPM apuntan a que la protección de la zona costera puede ayudar a restablecer poblaciones de moluscos afectadas por la extracción pesquera en los litorales rocosos. Sin embargo deben realizarse más estudios para apoyar esta hipótesis. En nuestro estudio la mayoría de las agrupaciones observadas fueron de abundancia baja ( $<10$ ind, Fig. 2). A pesar de la protección que por ley da el RNVSPHPM, sigue ocurriendo extracción artesanal de recursos pesqueros en la zona rocosa, por lo que la dominancia de agrupaciones de abundancia baja puede estar relacionada a esta extracción (E. Montero-Mata, comunicación personal, febrero 16, 2015; Ugalde, 2012).

La mayoría de agrupaciones (total y por cada categoría de abundancia) se dio en el sitio con menor vigilancia, contradiciendo el resultado esperado (Fig. 2). La distribución de $N$. scabricosta podría estar relacionada con factores como la exposición al oleaje, la disponibilidad de alimento, y el sustrato adecuado para asentamiento y refugios (Flórez-Jaramillo \& Cuéllar, 2013). Las interacciones de $N$. scabricosta con otras especies incluso pueden modificar la composición y estructura de la comunidad, por lo tanto esto también podría estar relacionado con la distribución observada (Levings \& Garrity, 1983).

La cantidad total de agregaciones disminuyó de la época seca a la lluviosa en el área de menor vigilancia, mientras que el área de mayor vigilancia mostró poca variación (Fig. 2). Entre la época seca y la época lluviosa, una fuerte marejada azotó la zona de estudio, con olas de hasta $2 \mathrm{~m}$ de altura y periodos de pico de ola de 24 s (O. Lizano, comunicación personal, octubre 24, 2017; MIO-CIMAR, 2017). 
Este evento pudo estar relacionado con la disminución de las agrupaciones de $N$. scabricosta (Fig. 2). En el sitio con menor vigilancia las agrupaciones de abundancia baja aumentaron, mientras que en el sitio con mayor vigilancia las agrupaciones de abundancia alta aumentaron (Fig. 2). Este patrón podría deberse a un mayor resguardo contra el estrés físico de la marejada debido a la conformación del litoral rocoso o a una menor extracción humana a corto plazo en el sitio con mayor vigilancia. Sin embargo, se deben realizar análisis más detallados para poder corroborar estas hipótesis.

Densidad de Tetraclita stalactifera: En Punta Mala la densidad promedio de $T$. stalactifera durante $2015\left(320 \pm 38 \mathrm{ind} / \mathrm{m}^{2}\right)$ fue mayor a los valores reportados para los litorales rocosos en el Golfo de Nicoya (Costa Rica): con promedios desde 29.6 a 160.0 ind/ $\mathrm{m}^{2}$ (Villalobos, 1980), pero estuvo dentro de lo reportado por Sutherland (1987) en Punta Mala (reportada como Tetraclita panamensis). Una de las razones por las cuales se ha encontrado una mayor densidad de $T$. stalactifera en Punta Mala comparado con el Golfo de Nicoya podría ser la influencia de agua dulce, ya que el Golfo de Nicoya presenta condiciones estuarinas (hasta 10.00 psu en algunas áreas internas) mientras que la zona de Punta Mala presenta salinidades marinas (31.86 psu) (Lizano, 2006; Lizano \& Vargas, 1994; MIO-CIMAR, 2017), sin embargo, esta hipótesis debe ser puesta a prueba. Se considera que la supervivencia de este tipo de organismos está relacionada con los puntos de surgencia, debido a que se presentan cambios en la temperatura del agua y en la disponibilidad de alimento (Menge, 2000; Rutllant \& Montecino, 2002). No obstante, la densidad de T. stalactifera también podría estar relacionada con la distribución y densidad de sus depredadores (Levings \& Garrity, 1983; Villalobos, 1980). Sutherland (1987) notó que varias poblaciones de $T$. stalactifera con una alta densidad se redujeron durante un monitoreo de tres años en Punta Mala, mientras en otras zonas intermareales siempre presentaron una baja densidad, pero esta no varió a lo largo del estudio, sin embargo no se encontró una causa directa para estas disminuciones.

Nuestros resultados no demostraron diferencias en la densidad de $T$. stalactifera entre el sitio con mayor vigilancia y el de menor vigilancia (Fig. 3). Es posible que otros factores, como la sedimentación, tengan una mayor influencia sobre la distribución de estos organismos sésiles (Littler, Martz, \& Littler, 1983). Tampoco se observaron cambios entre las épocas (Fig. 3), por lo que la marejada que se describió anteriormente aparentemente no tuvo un efecto sobre las densidades de T. stalactifera.

Macroalgas intermareales: En el sitio con menor vigilancia se observó una mayor cobertura de tapete de algas y una menor presencia de algas foliosas (rojas, pardas y verdes), comparado con el sitio con mayor vigilancia. El poblado de Esterillos Oeste es un sitio turístico donde las personas caminan frecuentemente por el litoral, mientras que el sitio con mayor vigilancia es menos transitado (E. Montero-Mata, comunicación personal, febrero 16, 2015). Por lo tanto, la menor cobertura de algas foliosas en el sitio con mayor acceso de turistas podría estar relacionada con el daño mecánico que provoca una persona al caminar sobre una zona rocosa (Davenport \& Davenport, 2006). La diferencia en cobertura entre ambos sitios también podría estar relacionada con la cantidad de nutrimentos o la presencia de contaminantes, debido a la cercanía con el pueblo, los cuales pueden afectar la composición de algas (Williams, Bracken, \& Jones, 2013). En general la cobertura del tapete de algas aumentó en los dos sitios para la época lluviosa. Es posible que la marejada que ocurrió entre ambas épocas afectara de forma negativa las algas foliosas que son susceptibles a la fuerza del oleaje y la entrada de sedimentos (Watanabe, Ito, Matsumoto, \& Arakawa, 2016).

Invertebrados bajo las rocas: Las diferencias observadas en la composición de la fauna bajo los cantos rodados entre el sitio con menor vigilancia y el sitio con mayor vigilancia podrían estar relacionadas con la mayor 
presencia de turistas en el sitio con menor vigilancia, sin embargo esta hipótesis debe ser comprobada. Es posible que algunas personas volteen rocas con mayor frecuencia en la zona con menor vigilancia, debido a la cercanía con el pueblo, lo que puede provocar la mortalidad de los organismos por daño mecánico (Davenport \& Davenport, 2006). Las actividades turísticas que no están reguladas y entran en contacto directo con los organismos marinos pueden afectar a los ecosistemas (Bravo, Márquez, Marzinelli, Mendez, \& Bigatti, 2015). Además, organismos como pepinos de mar y quitones fueron menos frecuentes en la zona de menor vigilancia, y ambos son utilizados para consumo humano (E. Montero-Mata, comunicación personal, febrero 16, 2015; Naranjo, 2010). Los funcionarios del Refugio mencionaron que tradicionalmente se han extraído de forma artesanal moluscos (e.g. Siphonaria gigas, Strombus galeatus, Malea ringens y Pinctada mazatlanica) y crustáceos (Panulirus gracilis). A pesar de que en años recientes ha disminuido esta actividad, aún persiste la extracción de recursos (Ugalde, 2012).

\section{AGRADECIMIENTOS}

Se agradece al SINAC por facilitar el personal (Randall Vargas, Andrés Jiménez, Liliana Rubí, Oscar Masis, Roger Madrigal, Luis Castro, Esteban Montero, Alonso Villalobos, Rodney Piedra, Arturo Rojas, Fabricio Álvarez, Paulino Valverde, Marco García, Álvaro Bermúdez) y las instalaciones del Refugio Nacional de Vida Silvestre Playa Hermosa-Punta Mala, para realizar el trabajo de campo. Se agradece a Esteban Montero Mata del Refugio de Vida Silvestre Playa Hermosa-Punta Mala por suministrar la información sobre los decomisos de extracción ilegal en la zona rocosa de Punta Mala. Se agradece a Leonardo Chacón y Davis Morera de la Universidad de Costa Rica por el apoyo logístico y durante el muestreo. Se agradece además a los revisores anónimos que con sus contribuciones ayudaron a mejorar la calidad de este trabajo. Se agradece a GIZ por los fondos suministrados por el contrato
83189134, PNUD con el proyecto Consolidación de las Áreas Marinas Protegidas de Costa Rica (CAMP) por financiar equipo para la recolección de datos, y pasos metodológicos de los que este trabajo forma parte. A la Vicerrectoría de Investigación con el proyecto \#808B5-802, Vicerrectoría de Acción Social con el proyecto \#ED-1242 y CIMAR de la UCR, por la capacidad institucional, el transporte y llevar a cabo el proyecto.

\section{RESUMEN}

Los intermareales rocosos son sitios importantes para peces, aves, invertebrados y algas entre otros, que se utilizan para la extracción de recursos alimenticios para las comunidades costeras. Se presentan los resultados de la evaluación de una costa rocosa en el Pacífico Central de Costa Rica (Refugio Nacional de Vida Silvestre Playa Hermosa-Punta Mala), usando indicadores biológicos propuestos por el protocolo del Ministerio de Ambiente y Energía. Se analizó la influencia de la vigilancia en dos sitios dentro de un AMP sobre el intermareal rocoso. La recolección de datos se realizó durante la época seca y la época lluviosa de 2015. Los datos mostraron que Nerita scabricosta presentó muchas colonias formadas por pocos individuos. La cobertura de macroalgas y los invertebrados bajo las rocas mostraron una leve diferencia entre los niveles de vigilancia. La densidad de Tetraclita stalactifera no mostró variaciones. Se sugiere la hipótesis que el daño mecánico asociado al turismo, pesca y extracción, está influyendo sobre los organismos de la zona rocosa. Algunos de los bioindicadores utilizados mostraron ser influenciados por los niveles de vigilancia y aplicación de las medidas de manejo.

Palabras clave: área marina protegida; bioindicador; macroalgas intermareales; Nerita scabricosta; Punta Mala; Tetraclita stalactifera; zona costera de Costa Rica.

\section{REFERENCIAS}

Aguilar, T. (1998). Protección del patrimonio geológico de Costa Rica. Revista Reflexiones, 69, 1-11.

Alvarado, J. J., Cortés, J., Esquivel, M. F., \& Salas, E. (2012). Costa Rica's marine protected areas: status and perspectives. Revista de Biología Tropical, 60, 129-142.

Bakus, G. J. (1968). Zonation in marine gastropods of Costa Rica and species diversity. The Veliger, 10, 207-211. 
Banks, S. A., \& Skilleter, G. A. (2002). Mapping intertidal habitats and an evaluation of their conservation status in Queensland, Australia. Ocean \& Coastal Management, 45(8), 485-509.

Barrantes, G., \& Pereira, A. (1992). Abundancia y fluctuaciones de aves limícolas (Charadriiformes) en una playa fangosa de Chomes, Costa Rica. Revista de Biología Tropical, 40(3), 303-307.

Bravo, G., Márquez, F., Marzinelli, E. M., Mendez, M. M., \& Bigatti, G. (2015). Effect of recreational diving on Patagonian rocky reefs. Marine Environmental Research, 104, 31-36.

Casu, D., Ceccherelli, G., Curini-Galletti, M., \& Castelli, A. (2006). Human exclusion from rocky shores in a mediterranean marine protected area (MPA): an opportunity to investigate the effects of trampling. Marine Environmental Research, 62(1), 15-32.

Clarke, K. R., \& Gorley, R. N. (2006). PRIMER V6: user manual-tutorial. Plymouth: Plymouth Marine Laboratory.

Crawley, M. J. (2007). The R Book. Chichester: John Wiley \& Sons.

Crowe, T. P., Thompson, R. C., Bray, S., \& Hawkins, S. J. (2000). Impacts of anthropogenic stress on rocky intertidal communities. Journal of Aquatic Ecosystem Stress and Recovery, 7, 273-297.

Davenport, J., \& Davenport, J. L. (2006). The impact of tourism and personal leisure transport on coastal environments: a review. Estuarine, Coastal and Shelf Science, 67(1-2), 280-292.

Defeo, O., McLachlan, A., Schoeman, D. S., Schlacher, T. A., Dugan, J., Jones, A., Lastra, M., \& Scapini, F. (2009). Threats to sandy beach ecosystems: a review. Estuarine, Coastal and Shelf Science, 81, 1-12.

Flórez-Jaramillo, L. Á., \& Cuéllar, J. L. (2013). Comportamiento circadiano del gasterópodo Nerita scabricosta en el intermareal rocoso de Isla La Palma, Pacífico Colombiano. Boletín de Investigaciones Marinas y Costeras-INVEMAR, 42(1), 173-179.

Hammer, Ø., Harper, D. A. T., \& Ryan, P. D. (2001). PAST: paleontological statistics software package for education and data analysis. Palaeontologia Electronica, 4(1), 1-9.

Krebs, C. J. (1999). Ecological Methodology. California: Addison - Wesley.

Lester, S. E., Halpern, B. S., Grorud-Colvert, K., Lubchenco, J., Ruttenberg, B. I., Gaines, S. D., Airamé, S., \& Warner, R. R. (2009). Biological effects within no-take marine reserves: a global synthesis. Marine Ecology Progress Series, 384, 33-46.
Levings, S. C., \& Garrity, S. D. (1983). Diel and tidal movement of two co-occurring neritid snails; differences in grazing patterns on a tropical rocky shore. Journal of Experimental Marine Biology and Ecology, 67(3), 261-278.

Little, C., \& Kitching, J. A. (1996). The Biology of Rocky Shores. New York: Oxford University Press.

Littler, M. M., Martz, D. R., \& Littler, D. S. (1983). Effects of recurrent sand deposition on rocky intertidal organisms: importance of substrate heterogeneity in a fluctuating environment. Marine Ecology Progress Series, 11, 129-139.

Lizano, O. (2006). Algunas características de las mareas en la costa Pacífica y Caribe de Centroamérica. Revista de Ciencia y Tecnología, 24, 51-64.

Lizano, O., \& Vargas, J. A. (1994). Distribución espaciotemporal de la salinidad y la temperatura en la parte interna del Golfo de Nicoya. Tecnología en Marcha, 12(2), 3-16.

Marliave, J. B. (1986). Lack of planktonic dispersal of rocky intertidal fish larvae. Transactions of the American Fisheries Society, 115(1), 149-154.

Mazé, R. A., Fuertes, B., \& Pena, J. C. (2006). Patrones de distribución de los peces intermareales de algunas pozas rocosas de la costa cantábrica (Norte de España). Boletín de La Real Sociedad Española de Historia Natural Sección Biológica, 101, 37-43.

Menge, B. A. (1991). Relative importance of recruitment and other causes of variation in rocky intertidal community structure. Journal of Experimental Marine Biology and Ecology, 146(1), 69-100.

Menge, B. A. (2000). Top-down and bottom-up community regulation in marine rocky intertidal habitats. Journal of Experimental Marine Biology and Ecology, 250, 257-289.

MIO-CIMAR. (2017). Módulo de Información Oceanográfica, Centro de Investigación en Ciencias del Mar y Limnología. Recuperado de http://miocimar.ucr.ac.cr

Murray, S. N., Ambrose, R., \& Dethier, M. N. (2006). Monitoring Rocky Shores. Londres: University of California Press.

Naranjo, H. (2010). Caracterización de los sistemas operacionales, modalidades y artes de pesca utilizados para la captura de la langosta Panulirus gracilis (Streets, 1871) en Guanacaste, Costa Rica. Revista Ciencias Marinas y Costeras, 2, 73-82.

Ortega, S. (1987). The effect of human predation on the size distribution of Siphonaria gigas (Mollusca: Pulmonata) on the Pacific of Costa Rica. Veliger, 29, 251-255. 
Ribeiro, P. D., Iribarne, O. O., Navarro, D., \& Jaureguy, L. (2004). Environmental heterogeneity, spatial segregation of prey, and the utilization of southwest Atlantic mudflats by migratory shorebirds. Ibis, 146(4), 672-682.

Riera, R., Pérez, Ó., Álvarez, O., Simón, D., Díaz, D., Monterroso, Ó., \& Núñez, J. (2016). Clear regression of harvested intertidal mollusks. A 20-year (19942014) comparative study. Marine Environmental Research, 113, 56-61.

Rojas, J., Villalobos, C. E., Chartier, F., \& Villalobos, C. R. (1988). Tamaño, densidad y reproducción de la barba de hacha, Tagelus peruvianus (Bivalvia: Solecurtidae) en el estero de Puntarenas, Costa Rica. Revista de Biología Tropical, 36(2B), 479-483.

RStudio Team. (2015). RStudio: Integrated Development for $R$. Boston: Rstudio Inc. Retrieved from www. rstudio.com

Rutllant, J., \& Montecino, V. (2002). Multiscale upwelling forcing cycles and biological response off northcentral Chile. Revista Chilena de Historia Natural, 75(217), e231.

Seyfried, H., Sprechmann, P., \& Aguilar, T. (1985). Sedimentología y paleoecología de un estuario del litoral Pacífico del Istmo Centroamericano primordial (Mioceno Medio, Costa Rica). Revista Geológica de América Central, 3, 1-68.

Sibaja-Cordero, J. A., Camacho-García, Y. E., \& VargasCastillo, R. (2014). Riqueza de especies de invertebrados en playas de arena y costas rocosas del Pacífico Norte de Costa Rica. Revista de Biología Tropical, 62(Supp. 4), 63-84.

Sibaja-Cordero, J. A., \& Martínez-Fernández, D. (2016) Protocolo PRONAMEC: Protocolo para el monitoreo ecológico de las playas rocosas. San José: PNUD-GEF.
Sutherland, J. P. (1987). Recruitment limitation in a tropical intertidal barnacle: Tetraclita panamensis (Pilsbry) on the Pacific coast of Costa Rica. Journal of Experimental Marine Biology and Ecology, 113(3), 267-282.

Sutherland, J. P., \& Ortega, S. (1986). Competition conditional on recruitment and temporary escape from predators on a tropical rocky shore. Journal of Experimental Marine Biology and Ecology, 95(2), 155-166.

Ugalde, J. (2012). Evaluación Ecológica Rápida (EER) de los Humedales del Refugio de Vida Silvestre Playa Hermosa -Punta Mala. San José: BIOMARCC-GIZ.

Venables, W. N., \& Ripley, B. D. (2013). Modern applied statistics with S-PLUS. Nueva York: Springer Science $\&$ Business Media.

Villalobos, C. R. (1980). Variations in population structure in the genus Tetraclita (Crustacea: Cirripedia) between temperate and tropical populations. III. $T$. stalactifera in Costa Rica. Revista de Biología Tropical, 28(1), 193-201.

Watanabe, H., Ito, M., Matsumoto, A., \& Arakawa, H. (2016). Effects of sediment influx on the settlement and survival of canopy-forming macrophytes. Scientific Reports, 6, 18677.

Williams, S. L., Bracken, M. E. S., \& Jones, E. (2013). Additive effects of physical stress and herbivores on intertidal seaweed biodiversity. Ecology, 94(5), 1089-1101.

Willis, S., \& Cortés, J. (2001). Mollusks of Manuel Antonio National Park, Pacific Costa Rica. Revista de Biología Tropical, 49(Suppl. 2), 25-36.

Withers, K., \& Chapman, B. R. (1993). Seasonal abundance and habitat use of shorebirds on an Oso Bay mudflat, Corpus Christi, Texas. Journal of Field Ornithology, 64(3), 382-392. 